\title{
Phytosphingosine promotes megakaryocytic differentiation of myeloid leukemia cells
}

\author{
Sang Hee Han ${ }^{1, \#}$, Jusong Kim ${ }^{1, \#}$, Yerim Her ${ }^{1}$, Ikjoo Seong ${ }^{1}$, Sera Park ${ }^{2}$, Deepak Bhattarai ${ }^{3}$, Guanghai Jin ${ }^{3}$, Kyeong Lee ${ }^{3}$, \\ Gukhoon Chung ', Sungkee Hwang ${ }^{4}$,Yun Soo Bae ${ }^{1, *}$ E Jaesang Kim ${ }^{1, *}$ \\ Departments of ${ }^{1}$ Life Science, ${ }^{2}$ Bio-informatics Science, Ewha Womans University, Seoul 03760, ${ }^{3}$ BK21 Plus R-FIND Team, College of \\ Pharmacy, Dongguk University, Goyang 10326, ${ }^{4}$ Doosan Corporation, Suwon 16229, Korea
}

\begin{abstract}
We report that phytosphingosine, a sphingolipid found in many organisms and implicated in cellular signaling, promotes megakaryocytic differentiation of myeloid leukemia cells. Specifically, phytosphingosine induced several hallmark changes associated with megakaryopoiesis from K562 and HEL cells including cell cycle arrest, cell size increase and polyploidization. We also confirmed that cell type specific markers of megakaryocytes, CD41a and CD42b are induced by phytosphingosine. Phospholipids with highly similar structures were unable to induce similar changes, indicating that the activity of phytosphingosine is highly specific. Although phytosphingosine is known to activate p38 mitogen-activated protein kinase (MAPK)-mediated apoptosis, the signaling mechanisms involved in megakaryopoiesis appear to be distinct. In sum, we present another model for dissecting molecular details of megakaryocytic differentiation which in large part remains obscure. [BMB Reports 2015; 48(12): 691-695]
\end{abstract}

\section{INTRODUCTION}

Megakaryocytes arise from hematopoietic stem cells and after terminal differentiation undergo apoptosis releasing platelets which are indispensable for hemostasis and thrombosis $(1,2)$. Megakaryocytic differentiation features stereotypical changes including cellular enlargement and polyploidization (1, 3). Expression of cell type specific surface markers including CD41a, CD61 and CD42b, is also a well-known phenomenon associated with the differentiation (3).

Although megakaryopoiesis through progressively more com-

*Corresponding authors. Yun Soo Bae, Tel: +82-2-3277-2729; Fax: +82-2-3277-3760; E-mail: baeys@ewha.ac.kr, Jaesang Kim, Tel: +822-3277-3414; Fax: +82-2-3277-3760; E-mail: jkim1964@ewha.ac.kr ${ }^{\#}$ These authors contributed equally to this work.

http://dx.doi.org/10.5483/BMBRep.2015.48.12.100

Received 21 May 2015, Revised 8 June 2015, Accepted 15 June 2015

Keywords: K562, Megakaryocyte, Phytosphingosine, Platelet, (R)TEMOSPho mitted precursors is well-defined at the cellular level, molecular mechanisms including signaling pathways and regulatory genes are far from clear. Cell signaling mediated by thromobopoietin (TPO) and c-mpl clearly plays a key role in induction of megakaryocytic differentiation and platelet genesis (4). However, down-stream signaling has hot been completely characterized yet. The slow progress is in large part due to the lack of a robust in vitro system to study the process. The primary hematopoietic stem cells are limited in supply as they cannot be renewed or expanded in vitro effectively.

Cell lines derived from myeloid leukemia including K562 and HEL have been useful in that they partly recapitulate the megakaryocyte differentiation in response to various signaling molecules $(5,6)$. For example, phorbol 12-myristate 13-acetate (PMA) can activate mitogen-activated protein kinase kinase-extracellular signal-regulated kinase (MEK-ERK) pathway and induce CD41a expression in response to AP1 activity from K562 cells (6). We have also reported that another molecule, 2-(Trimethylammonium) ethyl (R)-3-methoxy-3-oxo-2-stearamidopropyl phosphate ((R)-TEMOSPho) also induces megakaryocytic differentiation from K562 cells and primary human bone marrow-derived CD34 ${ }^{+}$cells (7).

Here, we present phytosphingosine as a novel differentiation inducer for megakaryocytes using K562 and HEL cells. Hallmark events including cell size increase, polyploidization and expression of CD41a and CD42b are confirmed. Importantly, although phytosphingosine is known to activate p38 MAPK signaling cascade-dependent apoptosis in myeloma cells including K562 cells (8), we provide evidences indicating that megakaryocytic differentiation is likely mediated by an alternate unknown pathway.

\section{RESULTS AND DISCUSSION}

We have previously reported that a phospholipid, (R)-TEMOSPho, induces megakaryocytic differentiation from K562 cells and primary CD34 ${ }^{+}$hematopoietic progenitor cells (7). We additionally screened diverse commercially available phospholipids (Fig. S1) to identify molecules with similar activities and identified phytosphingosine as a candidate based on induction of CD41a expression (Fig. 1A). Phytosphingosine was slightly but

ISSN: 1976-670X (electronic edition)

Copyright (C) 2015 by the The Korean Society for Biochemistry and Molecular Biology

(c) This is an open-access article distributed under the terms of the Creative Commons Attribution Non-Commercial License (http://creativecommons.org/licenses/by-nc/4.0) which permits unrestricted non-commercial use, distribution, and reproduction in any medium, provided the original work is properly cited. 


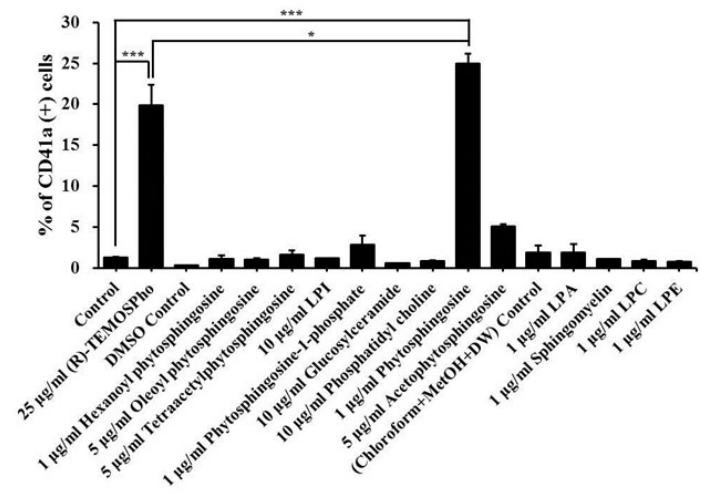

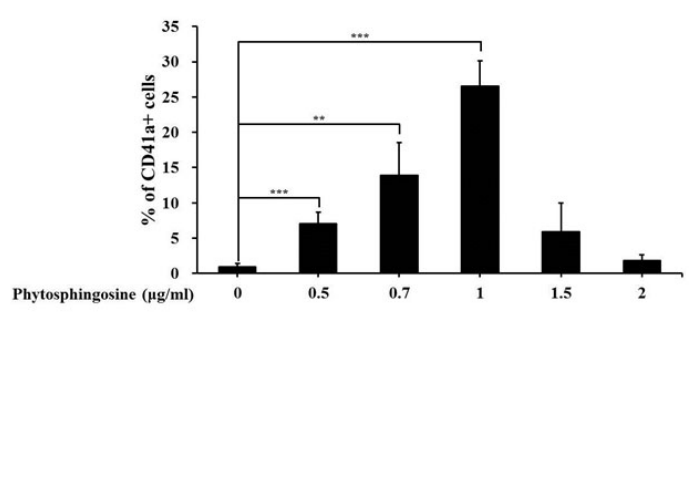

Fig. 1. Identification of phytosphingosine as a megakaryocytic differentiation inducing agent. (A) Induction of CD41a expression from K562 cells after 4 days of culture by phospholipids and sphingolipids at the indicated concentrations. Only phytosphingosine showed comparable activity to (R)-TEMOSPho. Results are averages \pm standard deviations of three independent assays. Statistical significance, tested by the Student's t-test is indicated. Typically, $10^{4}$ events were analyzed. (B) The induction of CD41a expression in K562 cells at different concentrations of phytosphingosine. Titration of phytosphingosine shows that induction of CD41a expression peaks at $1 \mu \mathrm{g} / \mathrm{ml}$ of phytosphingosine. Results are averages \pm standard deviations of four independent assays. Statistical significance, tested by the Student's $\mathrm{t}$-test is indicated $(* \mathrm{P}<0.05, * * \mathrm{P}<0.005, * * * \mathrm{P}<0.0005)$.

A

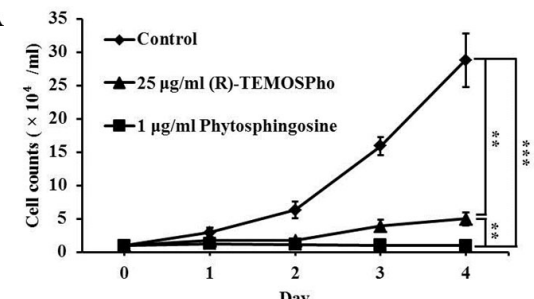

B

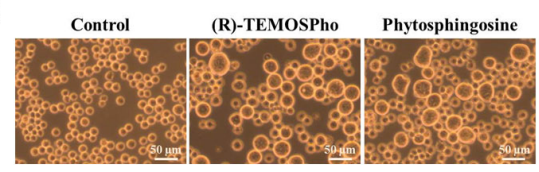

C

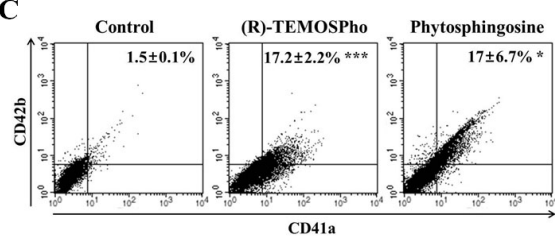

Fig. 2. Phytosphingosine-induced megakaryocytic differentiation of K562 cells. (A) Cell counts following treatment with $25 \mu \mathrm{g} / \mathrm{ml}$ (R)TEMOSPho or $1 \mu \mathrm{g} / \mathrm{ml}$ phytosphingosine. (B) Cell size increase after 4 days of treatment with (R)-TEMOSPho or phytosphingosine. Cells were visually examined and photographed by phase-contrast microscopy. Scale bars represent $50 \mu \mathrm{m}$. (C) Cell surface marker expression following treatment with (R)-TEMOSPho or Phytosphingosine for 6 days. The cells were labelled with monoclonal antibodies specific for the megakaryocyte cell surface markers CD41a and $\mathrm{CD} 42 \mathrm{~b}$ and analyzed by flow cytometry. Results are averages \pm standard deviations of three independent assays. Statistical significance, tested by the Student's t-test is indicated ( ${ }^{*} \mathrm{P}<0.05, * * \mathrm{P}<$ $0.005, * * * P<0.0005)$. reproducibly better than (R)-TEMOSPho in induction of CD41a expression. The inductive activity peaked at around $1 \mu \mathrm{g} / \mathrm{ml}$ of phytosphingosine (Fig. 1B), and apoptosis was evident beyond that level (Fig. S2) as has been reported for K562 cells (8).

Phytosphingosine treatment led to cell cycle arrest (Fig. 2A) (11) and concomitant enlargement (Fig. 2B), consistent with megakaryocytic differentiation. Furthermore, CD41a and CD42b were co-expressed in differentiating cells (Fig. 2C). Phytosphingosine showed more potent activity than (R)-TEMOSPho in inducing cell cycle arrest, but the two reagents showed comparable activity in inducing megakaryocytic differentiation (7).

We also examined polyploidization of K562 cells which accompanies typical megakaryopoiesis. Consistently, phytosphingosine treatment led to a significant rise in the cells with increased chromosomal contents (Fig. 3A). Specifically, proportions of cells with chromosomal content of $8 \mathrm{~N}$ increased at the cost of $2 \mathrm{~N}$ cells with (R)-TEMOSPho and phytosphingosine treatments. At the cellular level, polyploidy cells were identified by DAPI staining. Enlarged cells with multiple nuclei within single cell boundary were readily observed among cells treated with (R)-TEMOSPho and phytosphingosine but not among control cells (Fig. 3B).

The induction of megakaryocytic differentiation by phytosphingosine was also examined using another myelocytic leukemic cell line. Application of phytosphingosine to HEL cells led to limited but qualitatively similar changes in cell size, nuclear content and expression of megakaryocyte specific markers, indicating that the differentiation effect of phytosphingosine is not restricted to K562 cells (Fig. S3).

Next, we examined the signaling pathway which phytosphingosine activates during differentiation. It has been pre- 
viously reported that phytosphingosine induces apoptosis of K562 cells via activation of p38 MAPK pathway (8). In the study by Park and coworkers, $3 \mu \mathrm{g} / \mathrm{ml}$ of phytosphingosine was typically used to induce apoptosis. This is the level at which we also saw apoptosis (Fig. S2) while maximal differ-

A

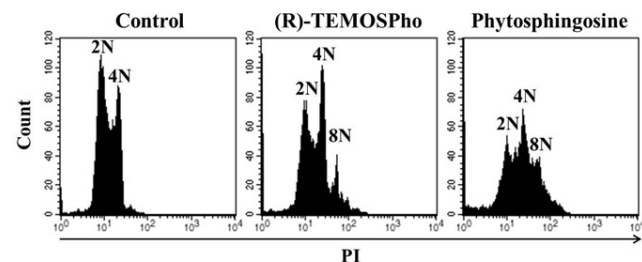

\begin{tabular}{|c|c|c|c|}
\hline & Control & (R)-TEMOSPho & Phytosphingosine \\
\hline $2 \mathrm{~N}$ & $55.9 \pm 6.6 \%$ & $39.6 \pm 5.8 \%$ & $29.5 \pm 7.5 \%$ \\
\hline $4 \mathrm{~N}$ & $39.6 \pm 3.7 \%$ & $44.8 \pm 4.0 \%$ & $37.2 \pm 2.8 \%$ \\
\hline$\geq 8 \mathrm{~N}$ & $0.7 \pm 0.2 \%$ & $12.3 \pm 1.7 \% * * *$ & $23.2 \pm 7.4 \% * *$ \\
\hline
\end{tabular}

B

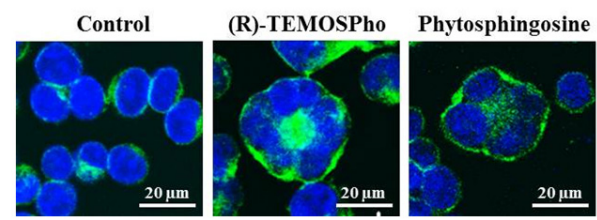

Fig. 3. Phytosphingosine-induced polyploidy of K562 cells. (A) Flow cytometric analyses for DNA contents following treatment with (R)-TEMOSPho or phytosphingosine for 4 days. Representative histograms are shown. The results from three independent assays are tabulated below. Statistical significance, tested by the Student's t-test is indicated ( $* * P<0.005, * * * P<0.0005)$. (B) K562 cells following treatment with (R)-TEMOSPho or phytosphingosine were DAPIstained (blue) for nuclei and F-actin-stained (green) with phalloidin-Alexa Fluor 488 to highlight the boundary of single cells. entiation was seen at $1 \mu \mathrm{g} / \mathrm{ml}$. We examined activation of phosphatidylinositol 3-kinase (PI3K)-Akt, p38, and ERK signaling (2). Only AKT showed enhanced phosphorylation at 1 $\mu \mathrm{g} / \mathrm{ml}$ phytosphingosine treatment (Fig. 4A). Next, we attempted to block PI3K-AKT signaling with the specific inhibitor LY294002 which at $1 \mu \mathrm{M}$ brought down phytosphingosine-mediated AKT activation near the basal level (Fig. 4B). Upon application of $5 \mu \mathrm{M}$ LY294002, the phosphorylation of AKT was virtually abrogated (Fig. 4B). Interestingly, induction of CD41a by phytosphingosine is not affected by LY294002 even at $5 \mu \mathrm{M}$ concentration (Fig. 4C). These data indicate that a pathway or pathways other than those examined mediate the induction of megakaryocytes by phytosphingosine.

In sum, we have demonstrated for the first time that phytosphingosine can induce differentiation of megakaryocytes from myeloid leukemia cells. Most notably, well-established changes associated with megakaryocytic differentiation including cell size increase, polyploidization and megakaryocyte specific marker expression were all demonstrated in K562 and HEL cell models. The effects on differentiation is comparable to that of (R)-TEMOSPho at least for K562 cells. Importantly, phytosphingosine is found as natural component of cells which implies that it may in fact be used as signaling molecule for megakaryocytic differentiation although this would require further investigations to demonstrate. Of note, it is likely that these two chemicals induce megakaryocytic differentiation via different mechanisms. Although PI3K-AKT pathway has been proposed to mediate the megakaryocytic induction by (R)TEMOSPho in K562 cells (7), we were not able to observe inhibition of phytosphingosine-mediated megakaryopoiesis by applying LY294002, a PI3K-AKT inhibitor. Of note, c-MPL, the TPO receptor, is not expressed in K562 cells (9). Taken together, phytosphingosine likely activates a novel pathway for the induction of megakaryocytic differentiation. It should be noted
$\mathbf{A}$

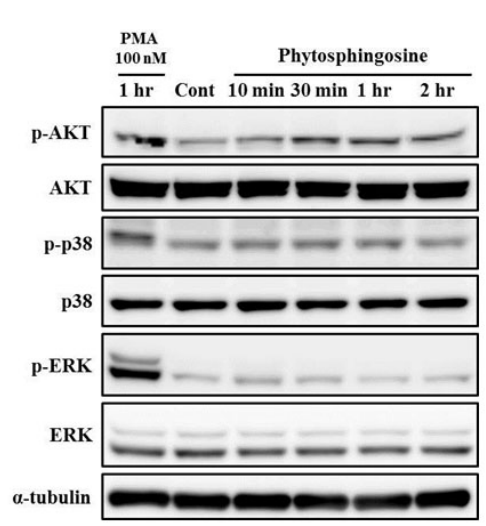

B

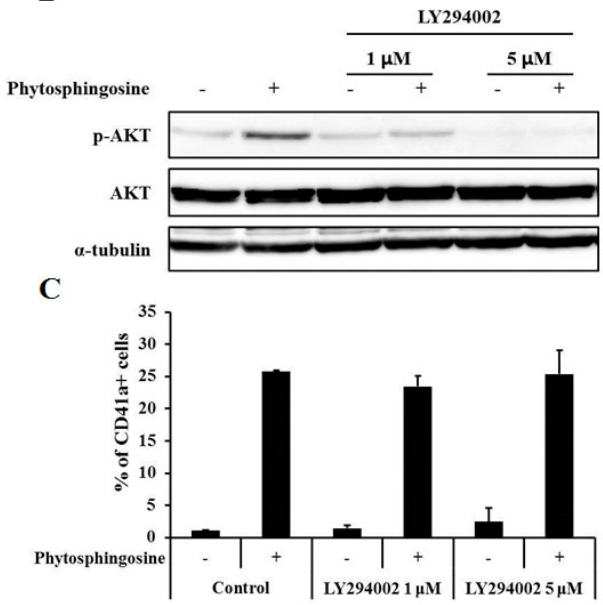

Fig. 4. Phytosphingosine-induced AKT phosphorylation is not involved in the megakaryocytic differentiation of K562 cells. (A) Immunoblot analyses were carried out using K562 cells treated with $1 \mu \mathrm{g} / \mathrm{ml}$ Phytosphingosine for the indicated times. PMA treatment was used as the positive control. Note the time dependent induction of AKT phosphorylation in K562 cells. (B) Inhibition of AKT activation by 1 hour pretreatment with LY294002 at the indicated concentrations. (C) K562 cells were differentiated with $1 \mu \mathrm{g} / \mathrm{ml}$ phytosphingosine in the absence or presence of LY294002. Note that \% of CD41a + cells is not altered by LY294002. 
that even in TPO or c-MPL knockout mice, low level megakaryocytes and platelets are found which implies that TPO-cMPL independent signaling pathway or pathways exist perhaps as back-up systems (3). Further molecular dissections, including determination of the down-stream signaling cascade, should lead to further understanding not only of the role of phytosphingosine but also of the mechanism of megakaryocytic differentiation.

\section{MATERIALS AND METHODS}

\section{Cell culture}

Routine K562 cell culture was carried out in RPMI 1640 medium containing $10 \%$ FBS. HEL cells were cultured in RPMI 1640 medium with $4.5 \mathrm{~g} / \mathrm{l}$ glucose, $10 \mathrm{mM}$ HEPES, and $1 \mathrm{mM}$ sodium pyruvate, supplemented with $10 \%$ FBS.

K562 and HEL cells were obtained from the American Type Culture Collection (Manassas, VA). RPMI 1640 medium and fetal bovine serum were purchased from Hyclone Laboratories Inc. (Logan, UT).

\section{Megakaryocyte differentiation}

Phospholipids and sphingolipids were dissolved in DMSO or alcohol-containing solution prior to application to cell culture media. For megakaryocytic differentiation, $3 \times 10^{4}$ cells were seeded in 6-well plate with $3 \mathrm{ml}$ of culture media which contained indicated levels of phytosphingosine or (R)-TEMOSPho. On subsequent days, $1 \mathrm{ml}$ of fresh media containing phytosphingosine or (R)-TEMOSPho was added to the culture media.

2-(Trimethylammonium) ethyl (R)-3-methoxy-3-oxo-2-stearamidopropyl phosphate ((R)-TEMOSPho) was synthesized following previously reported methods with modifications (10). Sphingomyelin was purchased from Sigma Aldrich (St. Louis, $\mathrm{MO}$ ), and Oleoyl phytosphingosine, Tetraacetyl phytosphingosine, Hexanoyl phytosphingosine, phytosphingosine, Acetyl phytosphingosine, Glucosyl cerarmide, Phosphatidyl choline were supplied by Doosan Biotech (Seoul, Korea). Phytosphingosine-1-phosphate, Lysophosphatidyl ethanolamine, Lysophosphatidyl choline, Lysophosphatidic acid, Lysophosphatidyl inositol were purchased from Avanti Polar Lipids, Inc. (Alabaster, $\mathrm{AL})$.

\section{Flow cytometry}

Cells were stained with $10 \mu \mathrm{l}(1 \mathrm{X})$ FITC conjugated antiCD41a antibody and/or PE-Cy5-conjugated anti-CD42b antibody and were analyzed by flow cytometry in a FACS Calibur BD Biosciences (San Jose, CA) using the BD Cell-Quest ${ }^{\mathrm{TM}}$ Pro version 6.0 software BD Biosciences (San Jose, CA). For DNA content and apoptosis analyses (12), cells were incubated for 25 minutes on ice in staining solution $(25 \mu \mathrm{g} / \mathrm{ml} \mathrm{Pl}, 0.1 \%$ saponin, $30 \mu \mathrm{g} / \mathrm{ml}$ RNase A in PBS pH 7.4) and analyzed by FACS Calibur.

FITC-conjugated anti-CD41a antibody and PE-Cy5-conjugated anti-CD42b antibody were purchased from BD Biosciences
(San Jose, CA, USA). Propidium iodide (PI) was from SigmaAldrich (St. Louis, MO). RNase A was purchased from USB (Cleveland, $\mathrm{OH}$ ).

\section{Phalloidin staining}

Cells were fixed and permeabilized with $1 \%$ paraformaldehyde in PBS containing $0.1 \%$ Triton X-100 for 1 hour at room temperature (RT). Subsequently, cells were blocked with $1 \%$ BSA in PBS containing Tween $20(0.1 \%)$ for 1 hour at RT and then stained for F-actin with phalloidin-Alexa Fluor 488 (1:200, Invitrogen, Carlsbad, CA) at RT for 1 hour. Cells were counterstained with 4'-6-Diamidino-2-phenylindole (DAPI) and visualized using a Zeiss laser scanning microscope (LSM 510 META, Zeiss, Germany) using a 40 x $/ 1.2 \mathrm{~W}$ apochromat objective (Zeiss, Germany).

Alexa 488-conjugated phalloidin was purchased from Invitrogen (Carlsbad, CA). 4'-6-Diamidino-2-phenylindole (DAPI) was from Sigma-Aldrich (St. Louis, MO).

\section{Westem blotting}

Whole-cell lysates $(50 \mu \mathrm{g})$ from K562 cells were subjected to $10 \%$ SDS-PAGE, transferred to nitrocellulose transfer membrane and incubated with primary antibodies overnight at $4^{\circ} \mathrm{C}$. Anti-p-AKT, anti-AKT, anti-p-Erk-1,2, anti-Erk-1,2, anti-p-p38 and anti-p38 antibodies were purchased from Cell Signalling Technology (Danvers, MA, USA) and used at a concentration of $1: 1,000$. After incubation with the secondary antibodies, blots were developed using an ECL immunodetection kit.

\section{ACKNOWLEDGEMENTS}

This study was supported by grants from the Ministry of Science, ICT and Future Planning via National Research Foundation (2012M3A9B4028785 to YSB and 2012M3A9B4028766 JK) and Redoxomics Grant (2012M3A9C5048708). Support also came from the Ministry of Health \& Welfare, Republic of Korea (A120262). SHH, GC, YSB and JK are listed as co-inventors of a patent application pertaining to this work.

\section{REFERENCES}

1. Kaushansky K (2008) Historical review: megakaryopoiesis and thrombopoiesis. Blood 111, 981-986

2. Severin S, Ghevaert C and Mazharian A (2010) The mitogen-activated protein kinase signaling pathways: role in megakaryocyte differentiation. J Thromb Haemost 8, 1726

3. Deutsch VR and Tomer A (2006) Megakaryocyte development and platelet production. Br J Haematol 134, 453-466

4. Kaushansky K and Drachman JG (2002) The molecular and cellular biology of thrombopoietin: the primary regulator of platelet production. Oncogene 21, 3359-3367

5. Levay K and Slepak VZ (2007) Tescalcin is an essential factor in megakaryocytic differentiation associated with Ets family gene expression. J Clin Invest 117, 2672-2683 
6. Jalagadugula G, Dhanasekaran DN, Kim S, Kunapuli SP and Rao AK (2006) Early growth response transcription factor EGR-1 regulates Galphaq gene in megakaryocytic cells. J Thromb Haemost 4, 2678-2686

7. Limb JK, Song D, Jeon M et al (2015) 2-(Trimethylammonium)ethyl (R)-3-methoxy-3-oxo-2-stearamidopropyl phosphate promotes megakaryocytic differentiation of myeloid leukaemia cells and primary human $\mathrm{CD} 34(+)$ haematopoietic stem cells. J Tissue Eng Regen Med 9, 435-446

8. Park MT, Kang YH, Park IC et al (2007) Combination treatment with arsenic trioxide and phytosphingosine enhances apoptotic cell death in arsenic trioxide-resistant cancer cells. Mol Cancer Ther 6, 82-92
9. Columbyova L, Loda M and Scadden DT (1995) Thrombopoietin receptor expression in human cancer cell lines and primary tissues. Cancer Res 55, 3509-3512

10. Kim YA, Chung HM, Park JS et al (2003) Synthesis of novel lysophosphatidylcholine analogues using serine as chiral template. J Org Chem 68, 10162-10165

11. Kim S and Kang H (2013) miR-15b induced by platelet-derived growth factor signaling is required for vascular smooth muscle cell proliferation. BMB Rep 46, 550-554

12. Zhou C, Shen Q, Xue J, Ji C and Chen J (2013) Overexpression of TTRAP inhibits cell growth and induces apoptosis in osteosarcoma cells. BMB Rep 46, 113-118 